\title{
Comparison of two methods for measuring the pubococcygeal line from sagittal- plane magnetic resonance imaging
}

Stéphanie Madill,

An Tang,

Stéphanie Pontbriand-Drolet,

Chantal Dumoulin

First published: 29 June 2011

Linda Brubaker led the review process.

Conflict of interest: none.

\begin{abstract}
Aims

The pubococcygeal line (PCL) is an important reference line for determining measures of pelvic organ support on sagittal-plane magnetic resonance imaging (MRI); however, there is no consensus on where to place the posterior point of the PCL. As coccyx movement produced during pelvic floor muscle (PFM) contractions may affect other measures, optimal placement of the posterior point is important. This study compared two methods for measuring the PCL, with different posterior points, on T2-weighted sagittal MRI to determine the effect of coccygeal movement on measures of pelvic organ support in older women.
\end{abstract} Methods

MRI of the pelvis was performed in the midsagittal plane, at rest and during PFM contractions, on 47 community-dwelling women 60 and over. The first PCL was measured to the tip of the coccyx (PCLtip) and the second to the sacrococcygeal joint (PCLjnt). Four measures of pelvic organ support were made using each PCL as the reference line: urethrovesical junction height, uterovaginal junction height, $\mathrm{M}$-line and levator plate angle.

Results

During the PFM contraction the PCLtip shortened and lifted $(P<0.001)$; the PCLjnt did not change $(P>0.05)$. The changes in the four measures of pelvic organ support were smaller when measured relative to the PCLtip as compared to those to the PCLjnt $(P<0.001)$.

Conclusions

Coccyx movement affected the length and position of the PCLtip, which resulted in underestimates of the pelvic-organ lift produced by the PFM contraction. Therefore, we recommend that the PCL be measured to the sacrococcygeal joint and not to the tip of the coccyx.

\section{INTRODUCTION}

The pubococcygeal line (PCL) is one of the primary landmarks used to assess pelvic organ support on sagittal-plane magnetic resonance imaging (MRI) of the pelvis. In the literature, there is agreement on using the inferior edge of the pubic symphysis as the anterior landmark; however, there is currently no consensus on where to place the posterior point of the PCL. 1 Various authors have placed it at the tip of the coccyx, $\underline{2}$ the last vertical or horizontal joint of the coccyx $\underline{\underline{3}-5}$ or the sacrococcygeal joint. $\underline{6}$ To add to the confusion, when the line is drawn to the sacrococcygeal joint it is also referred to as the sacrococcygealinferior pubic point line (SCIPP line). $\underline{7-11}$

Different placements not only make it difficult to compare results between studies, but may also introduce error into the measurements. $\mathrm{B} \emptyset$ et al.12 found that the coccyx moves ventrally during a pelvic floor muscle (PFM) contraction and dorsally during straining. Thus, coccyx movement is likely to affect the length and 
position of the PCL when it is measured to the tip of the coccyx. The resulting changes would thus render comparisons of pelvic organ support between conditions of rest, PFM contraction and straining less valid. Therefore, the primary goal of this study was to compare the lengths of two PCLs, each measured to different posterior ends: the tip of the coccyx (PCLtip) and the sacrococcygeal joint (PCLjnt), under three conditions: rest, PFM maximum voluntary contraction (MVC) and straining, to determine which of the two PCLs provides the most valid baseline for measures of pelvic organ support. These two posterior landmarks were chosen because they are at opposite ends of the coccyx and are, therefore, most likely to demonstrate any effects of coccyx movement. The second objective was to describe the effect of changes in the PCLs' lengths on four measures of pelvic organ support that use the PCL as a reference line: the urethrovesical junction height, the uterovaginal junction height, the M-line, and the levator plate angle.

\section{MATERIALS AND METHODS}

Women 60 years and older were recruited using advertisements in a local newspaper for seniors and through referrals from participating physicians. Interested women were screened by telephone and included in the study if they were independently ambulatory and were either continent (no report of any urine leakage for at least 1 year) or reported at least one episode weekly of mixed or stress urinary incontinence (MUI or SUI) over the previous 3 months. Women were excluded if they reported any contraindication to MRI, had symptoms consistent with other types of incontinence according to the Urogenital Distress Inventory, $\underline{13}$ reported conditions that might impair their ability to participate in the study or were taking medications known to affect continence. Women who passed the screening and were interested in participating in the study were mailed the consent form to read prior to their appointments. The study received ethical approval from the research ethics boards at the Centre de recherche de l'institut universitaire de gériatrie de Montréal, where data collection took place, and at each of the hospitals from which participants were recruited. The consent form was reviewed with each participant and written consent was obtained prior to the physical evaluation.

This study was part of a larger prospective cohort study with the objective to characterize and compare the morphology and function of the female PFM using MRI and dynamometry in women with and without incontinence aged 60 and over. This study was developed in order to ensure the validity of our measures of pelvic organ support prior to using these measures to assess changes in pelvic organ support produced by the training exercises and to entering these variable in the predictive model. All of the data for this study were collected at the pre-intervention evaluation, which also included an evaluation of pelvic floor muscle function using dynamometry and electromyography.

Using intra-vaginal palpation, dynamometry, electromyography and verbal feedback, an experienced pelvic floor physiotherapist taught the participants to perform PFM contractions correctly. They were then oriented to the MRI suite and the testing procedure, and asked to empty their bladders.

Magnetic resonance imaging in the sagittal plane was performed with a Siemens 3.0T Magnetom Trio, using an IPAT torso/pelvis coil centered at the symphysis pubis. First, 20 high-resolution slices were recorded at rest with T2 weighted fast spin echo sequences (field of view $24 \mathrm{~cm} \times 24 \mathrm{~cm}$, matrix $512 \times 256$, $5 \mathrm{~mm}$ thick/1 mm gap, TR 4,190 msec, TE $134 \mathrm{msec}$, Bandwidth $130 \mathrm{~Hz} /$ pixel, NEX 1, scan duration $146 \mathrm{sec})$. Then, during the PFM MVC and the straining manoeuvre, six acquisitions were made in the midsagittal plane with T2 weighted single-shot fast spin echo sequences to provide a cine view (field of view $24 \mathrm{~cm} \times 24 \mathrm{~cm}$, matrix $256 \times 256,6 \mathrm{~mm}$ thick $/ 0 \mathrm{~mm}$ gap, TR $=3,000 \mathrm{msec}$, TE $110 \mathrm{msec}$, Bandwidth $320 \mathrm{~Hz} /$ pixel, NEX 1, scan duration $18 \mathrm{sec}$ ). The PFM MVC instructions were: "Contract your muscles as hard as you can, as if you were holding back urine or gas." Effort during straining was controlled by having the volunteers blow into a standardized (Guillarme's) tube. The instructions were: "Blow into the tube and push as if you were passing stool."

The measurements at rest were taken from the midsagittal slice. For the PFM MVC and the straining images, the measurements were taken from the midsagittal slices that demonstrated the greatest bladder neck elevation and descent, respectively. The PCLtip was drawn from the inferior edge of the pubic symphysis to the tip of the coccyx and the PCLjnt was drawn from the inferior edge of the pubis symphysis to the anterior aspect of the sacrococcygeal joint line (see Fig. 1). The four measures of pelvic organ 
support were taken next. The height of the urethrovesical junction was measured as the perpendicular distance between the PCL and the anterior angle between the bladder and the urethra. 14 The height of the uterovaginal junction was measured as the perpendicular distance between the PCL and the anterior angle where the uterus flexes on the vagina.15 In women who had had hysterectomies, the superior-most point of the vagina was used as the landmark. The H-Line was measured from the inferior edge of the pubis symphysis to the posterior aspect of the rectum at the anorectal angle. 4 The M-line was measured as the perpendicular distance from the PCL to the posterior end of the H-line (i.e., at the anorectal angle). The levator plate angle was measured between the PCL and the H-line 3 (see Fig. 1). The PCLtip and its corresponding measures of pelvic organ support were performed first and then erased prior to measuring the PCLjnt and its corresponding measures of pelvic organ support.

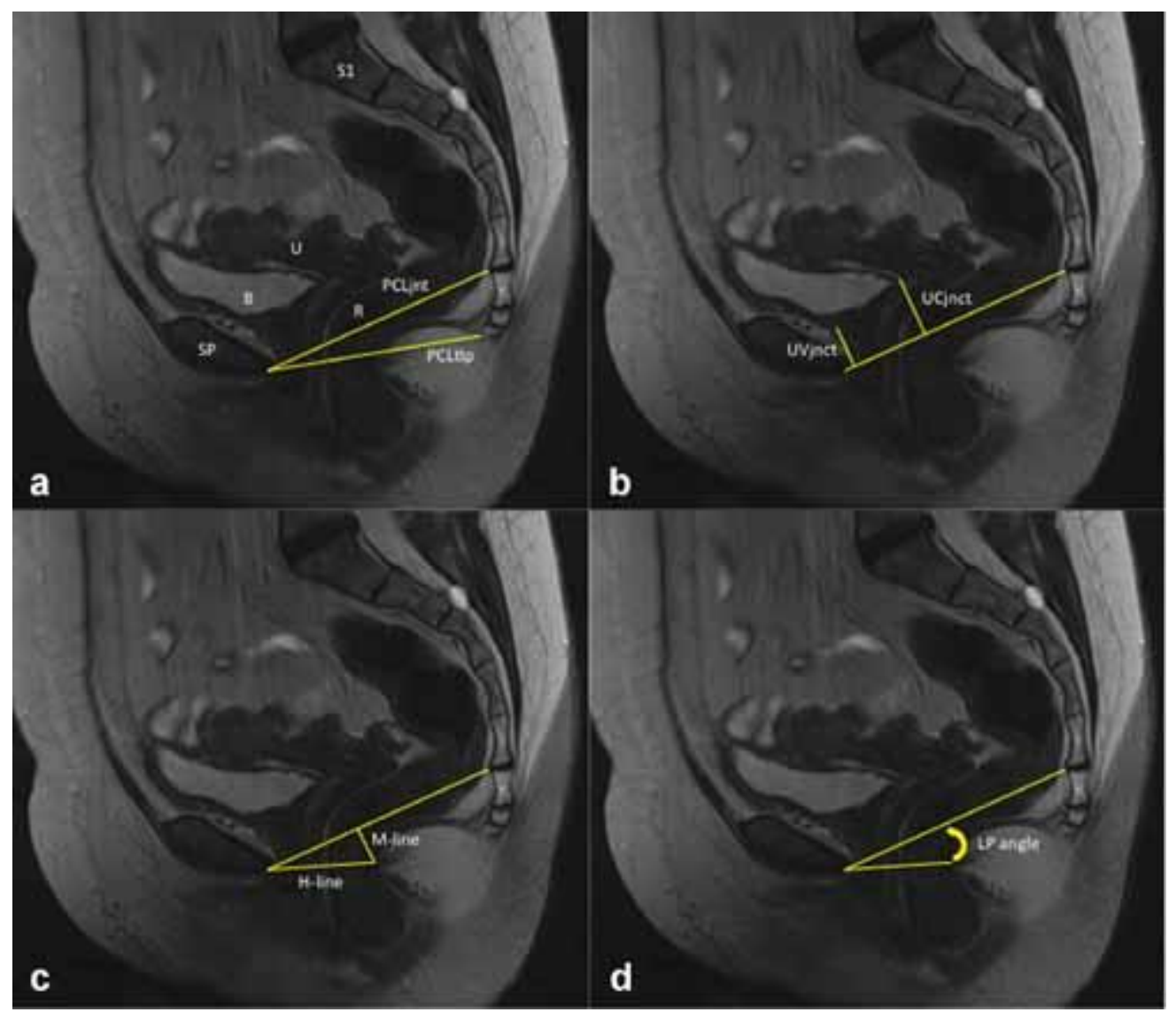

\section{Figure 1.}

Pubococcygeal lines and measures of pelvic organ support. a: The pubococcygeal lines were drawn from the inferior pubic symphysis anteriorly to the tip of the coccyx (PCLtip) and sacrococcygeal joint (PCLjnt). b: Urethrovesical junction (UVjnct) height and uterovaginal junction (UCjnct) height. c: M-line and (d) Levator plate (LP) angle. Each measure was taken twice on each image: once with the PCLtip as the baseline and a second time using the PCLjnt. For clarity, the images (b-d) are only shown with the PCLjnt as the baseline. SP, symphysis pubis; B, bladder; U, uterus; R, rectum; S1, first sacral vertebral body.

In order to test the intra-rater reliability of each measurement under the three conditions (rest, PFM MVC, and straining), images from 10 individuals were chosen at random: then, working independently, two raters (SJM and SPD) produced two sets of measurements for each image. The measurements were made on all of the images in the group once before being repeated. The intra-class correlations (ICCs), coefficients of variation $(\mathrm{CVs})$ and standard errors of the mean (SEMs) were calculated between each evaluator's first and 
second set of measurements for both of the PCLs under each of the conditions (rest, PFM MVC, and straining).

In order to test the inter-rater reliability of the measurements, 10 other participants were randomly chosen and their images analyzed; working independently, the two raters produced one set of measurements from each image. The ICCs, CVs, and SEMs were then calculated between the two evaluators' measurements of the 20 participants' images: the 10 that were measured once each by the 2 raters and 10 from the intra-rater reliability tests. Only the second set of measurements from the intra-rater reliability tests were used to remove possible learning effects. After confirming the intra- and inter-rater reliabilities, the images from the remaining volunteers were randomly assigned to the two evaluators, who took one set of measurements for each image.

To compare the two methods for measuring the PCL, the lengths of the two PCLs during the three conditions (rest, PFM MVC, and straining) were compared using a two-way repeated measures analysis of variance (ANOVA). The line by condition interaction was included in the model. Post hoc comparisons were made using the Bonferroni method to correct for multiple comparisons. As well, ICCs and BlandAltman plots were calculated by PCL for each pair of tasks.

The position of the PCLtip relative to the PCLjnt was assessed by measuring the angle between the two PCLs in each of the three conditions. These angles were then compared using a repeated measures ANOVA and post hoc comparisons were made using the Bonferroni method.

The two-way repeated measures ANOVA model was used to test for differences in the four measures of pelvic organ support (urethrovesical junction height, uterovaginal junction height, M-line, and levator plate angle) produced by changes in the length of the PCL between the conditions and post hoc comparisons were made using the Bonferroni method. Significance was set at $\alpha<0.05$ for all tests.

\section{RESULTS}

Forty-seven women participated: 14 continent, 24 with MUI, and 9 with SUI. As there were no differences among the continence groups in terms of age, parity, or body mass index (BMI), they are presented as aggregate data, mean (standard deviation): age $67.6( \pm 4.7)$ years, parity $1.7( \pm 1.5)$ births and BMI 25.5 $( \pm 3.9) \mathrm{kg} / \mathrm{m}^{2}$.

The PCL measurements demonstrated excellent intra- and inter-rater reliabilities for all three conditions. There were no differences in the intra- and inter-rater reliabilities between the PCLjnt and the PCLtip. The other measures of pelvic organ support also demonstrated good to excellent intra- and inter-rater reliabilities (see Table I). 
Table I. Intra- and Inter-Rater Reliabilites for the Sagittal-Plane MRI Measurements: Pubococcygeal Line to the Tip of the Coccyx (PCLtip), Pubococcygeal Line to the Sacrococcygeal Joint (PCLjnt), Height of the Urethrovesical Junction (UVjnct), Height of the Uterovaginal Junction (UCjnct), M-Line and Levator Plate Angle (LP Angle)

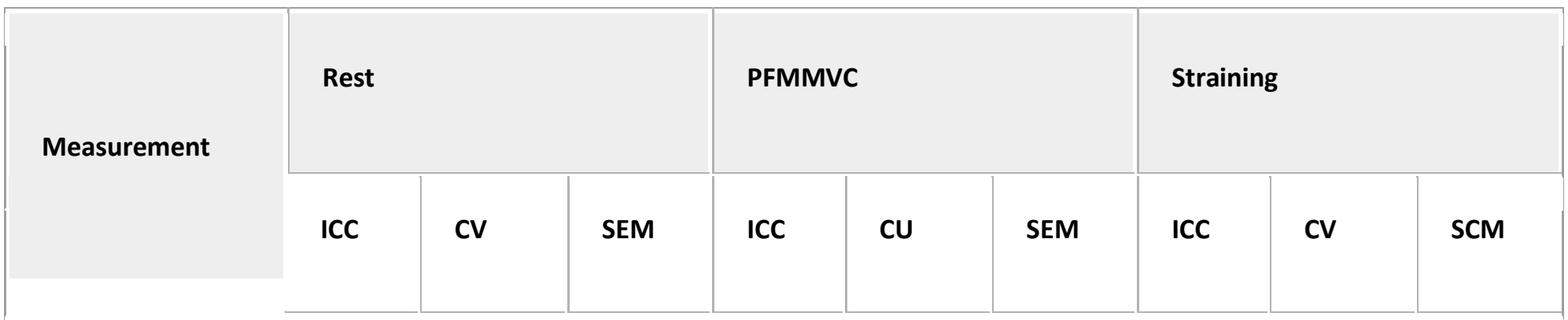

Each measurement was taken for three conditions: rest, pelvic floor muscle maximum voluntary contraction (PFM MVC) and straining, by two raters (SJM and SPD). ICC, intraclass correlation; CV, coefficient of variation (\%); and SEM, standard error of the mean (mm, except LP angle ${ }^{\circ}$ ).

PCLtip

Intra-rater

SJM

0.96

8.58

0.64

0.96

6.15

0.55

0.80

7.95

1.55

SPD

0.89

6.04

0.88

0.80

6.19

1.25

0.90

7.71

1.12

Inter-rater

$0.72 \quad 9.05$

1.76

0.79

8.82

1.52

0.82

9.36

1.52

PCLjnt

Inter-rater 


\begin{tabular}{|c|c|c|c|c|c|c|c|c|c|}
\hline SJM & 0.99 & 9.80 & 0.38 & 0.99 & 10.13 & 0.55 & 0.97 & 13.00 & 1.04 \\
\hline SPD & 0.99 & 9.11 & 0.43 & 0.97 & 9.84 & 0.84 & 0.98 & 9.71 & 0.68 \\
\hline Intra-rater & 0.96 & 7.85 & 0.92 & 0.96 & 8.01 & 0.91 & 0.97 & 7.96 & 0.75 \\
\hline \multicolumn{10}{|l|}{ UVjnct } \\
\hline \multicolumn{10}{|l|}{ Intra-rater } \\
\hline SJM & 0.95 & 53.81 & 0.68 & 0.96 & 24.84 & 0.39 & 0.95 & 32.80 & 1.18 \\
\hline SPD & 0.79 & 56.70 & 1.50 & 0.86 & 24.60 & 0.76 & 0.98 & 24.50 & 0.57 \\
\hline Inter-rater & 0.95 & 51.20 & 0.66 & 0.92 & 30.54 & 0.52 & 0.98 & 25.13 & 0.58 \\
\hline \multicolumn{10}{|l|}{ UCJnct } \\
\hline \multicolumn{10}{|l|}{ Inter-rater } \\
\hline SJM & 0.94 & 53.18 & 1.32 & 0.98 & 23.64 & 0.36 & 0.92 & 20.38 & 2.08 \\
\hline \multirow[t]{2}{*}{ SPD } & 0.97 & 50.85 & 0.84 & 0.95 & 23.65 & 0.75 & 0.98 & 18.12 & 1.24 \\
\hline & 0.98 & 59.24 & 0.90 & 0.98 & 29.42 & 0.62 & 0.95 & 17.91 & 1.93 \\
\hline
\end{tabular}


M-line

Inter-rater

SJM

SPD

Inter-rater

0.94

43.75

1.15

0.95

89.22

0.96

0.91

39.89

1.64

LP angle

Inter-rater

SJM

0.91

41.07

1.06

0.87

67.21

1.69

0.83

32.06

1.54

SPD

0.88

39.08

1.39

0.85

69.94

1.15

0.83

36.80

1.88

Inter-rater
0.76

16.88
0.89

17.11

0.36

0.91

49.45

0.67

The PCLjnt was longer than the PCLtip for all three conditions $(P<0.001)$. The line by condition interaction was significant $(P=0.027)$; the PCLjnt length did not change among the three conditions, while the PCLtip shortened during the PFM MVC. However, the shortening effect was small making the post hoc comparisons non-significant.

The analyses were repeated for the 31 cases $(66 \%)$ that demonstrated at least a $2 \mathrm{~mm}$ difference in PCLtip length between rest and either the PFM MVC or straining condition (the excluded cases included three cases that demonstrated $<2 \mathrm{~mm}$ change and 13 cases that demonstrated no change in PCLtip length among the conditions). This subgroup did not differ significantly in age, parity, or BMI from the participants as a whole. The line by condition interaction was again significant $(P<0.001)$ and the same shortening pattern was demonstrated. In the post hoc analyses, the PCLtip was shorter during the PFM MVC than it was during the rest or straining conditions $(P<0.001$ and $P=0.004$, respectively), and there was no difference in the length of the PCLtip between rest and straining $(P=0.84)$. The lack of difference between the rest 
and straining conditions seems to be due to the observation that the participants demonstrated two distinct patterns of movement during straining: in 18 of the 31 women demonstrating coccyx movement, the coccyx extended on the sacrum resulting in a longer PCLtip, while the remaining 13 demonstrated flexion of the coccyx on the sacrum resulting in a shortening of the PCLtip. Again, there was no change in the length of the PCLjnt with any of the manoeuvres ( $P>0.84$ for all comparisons) in this subgroup (see Fig. 2).
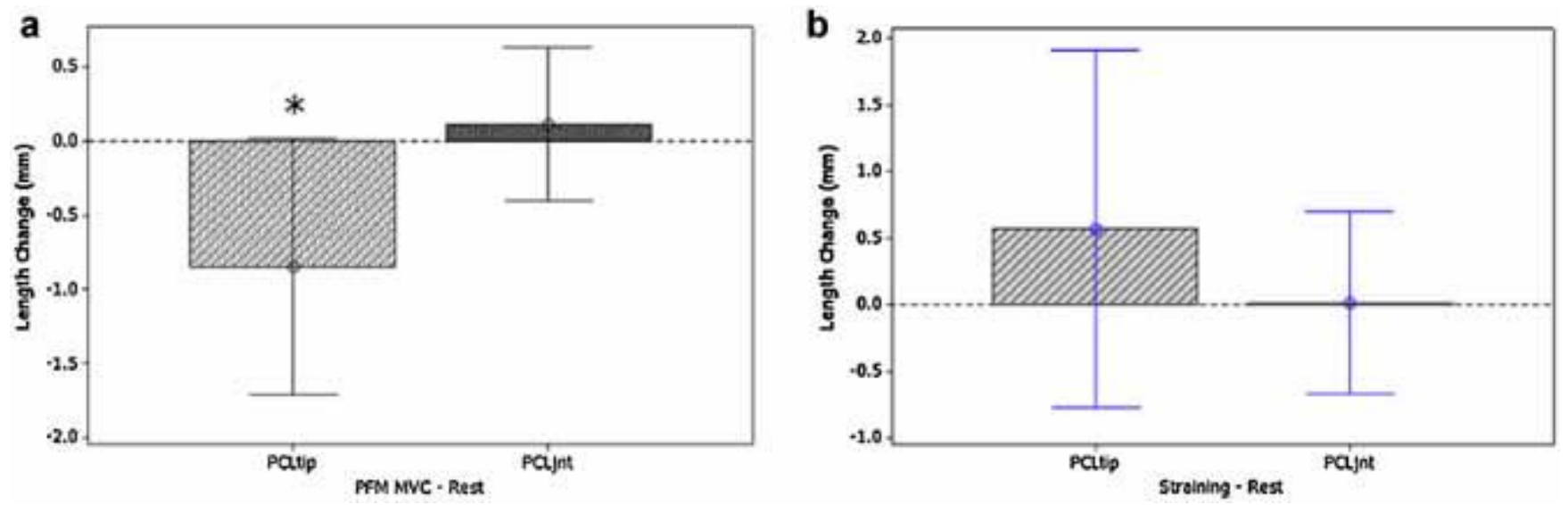

Figure 2.

Change in PCL length from rest to (a). Pelvic floor muscle maximum voluntary contraction (PFM MVC) and (b) Straining. Changes that differ significantly from 0 are indicated by $* P<001$.

The ICCs were excellent for both PCLs (PCLtip $\geq 0.84$ and PCLjnt $\geq 0.97$ ); however, the ICCs were significantly higher for the PCLjnt as compared to the PCLtip (demonstrated by no overlap between the 95\% confidence intervals). The Bland-Altman plots for the PCLtip all demonstrated greater variability as compared to the plots for the PCLjnt (see Fig. $\underline{3}$ ). 

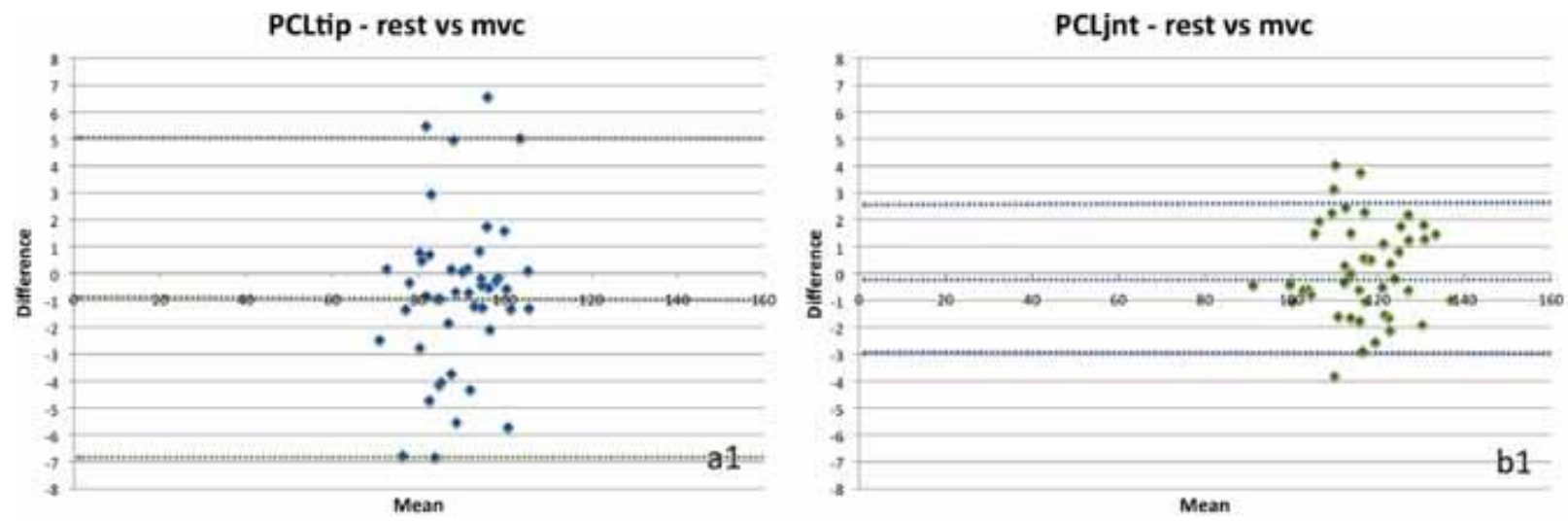

PLCtip mvc vs straining
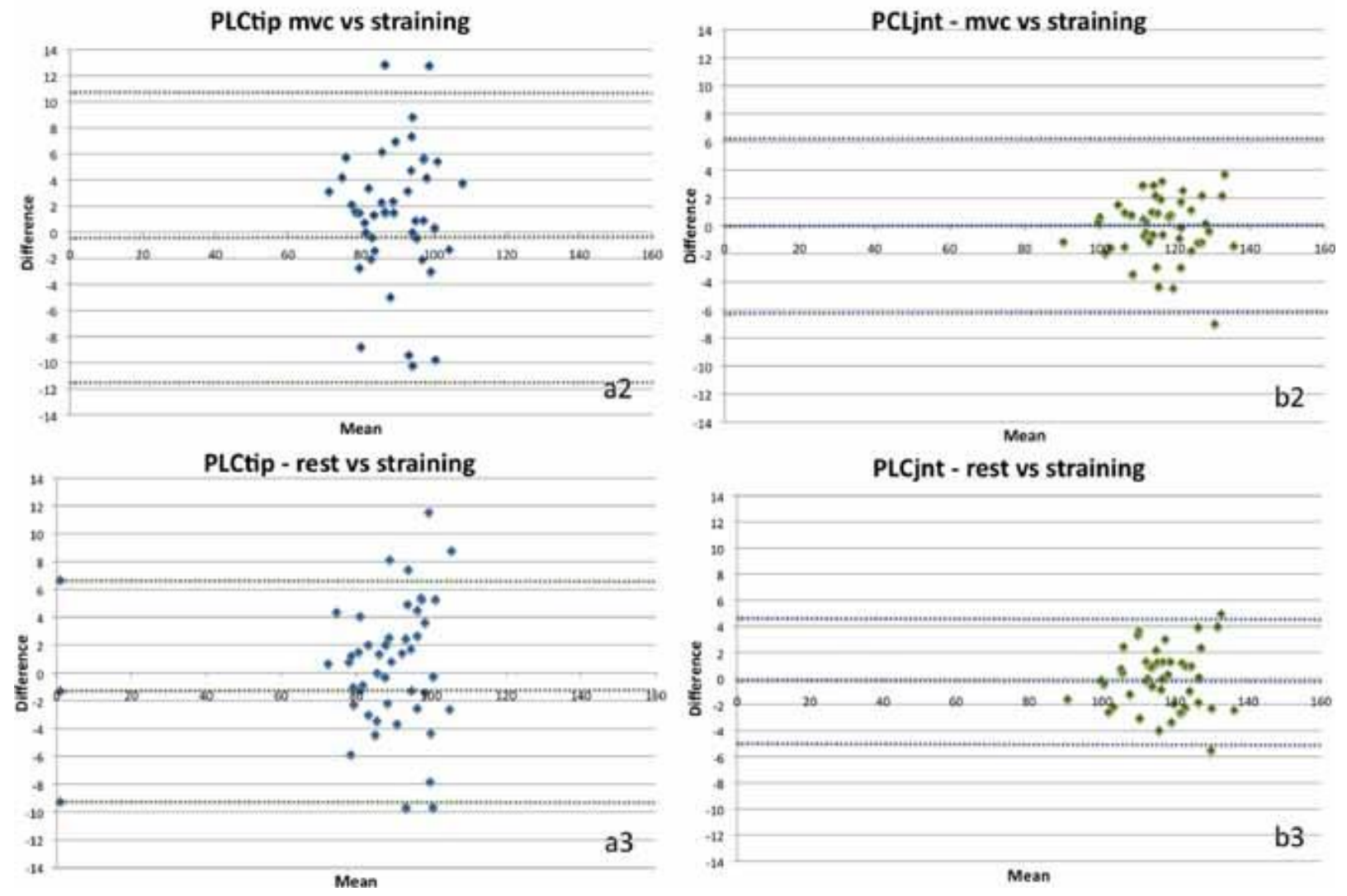

Figure 3.

Bland-Altman plots by pubococcygeal line between conditions. a: Pubococcygeal line to the tip of the coccyx (PCLtip), (b) pubococcygeal line to the sacrococcygeal joint (PCLjnt), (1) Rest versus maximum voluntary pelvic floor muscle contraction (PFM MVC), (2) PFM MVC versus straining, and (3) rest versus straining. The dotted lines indicate the overall mean and two standard deviations.

The position of the PCLtip relative to the PCLjnt also changed among the conditions. At rest the mean angle between the two PCLs was $9.88^{\circ}( \pm 4.14)$, during the PFM MVC the PCLtip moved towards the PCLjnt and the mean angle decreased to $7.48^{\circ}( \pm 4.08)$, and during straining the mean angle between the two PCLs was $10.10^{\circ}( \pm 4.21)$. The angle between the two PCLs was significantly different during the PFM MVC than it was at rest and during straining $(P<0.001$ for both). The angle between the two PCLs during straining was not significantly different from the angle at rest $(P=0.46)$, again probably because of the two patterns of coccyx movement demonstrated by the subgroup. 
As the PCL is used as a baseline from which to make several measures of pelvic organ support (e.g., the heights of the urethrovesical and the uterovaginal junctions, the length of the M-line and as one arm of the levator plate angle), it is clinically useful to know how changes in the PCLtip's length and position, during the different conditions, affect these measures. For all four measures, the changes between the rest and PFM MVC conditions were significantly different between those made with the PCLtip as the baseline and those made with the PCLjnt as the baseline $(P<0.001$ for each comparison). The changes in the four measurements between the rest and straining conditions were not significantly different depending on which PCL was used as the baseline ( $P>0.22$ for each comparison). Again the two patterns of coccyx movement seen during straining probably account for this lack of difference (see Fig. 4).
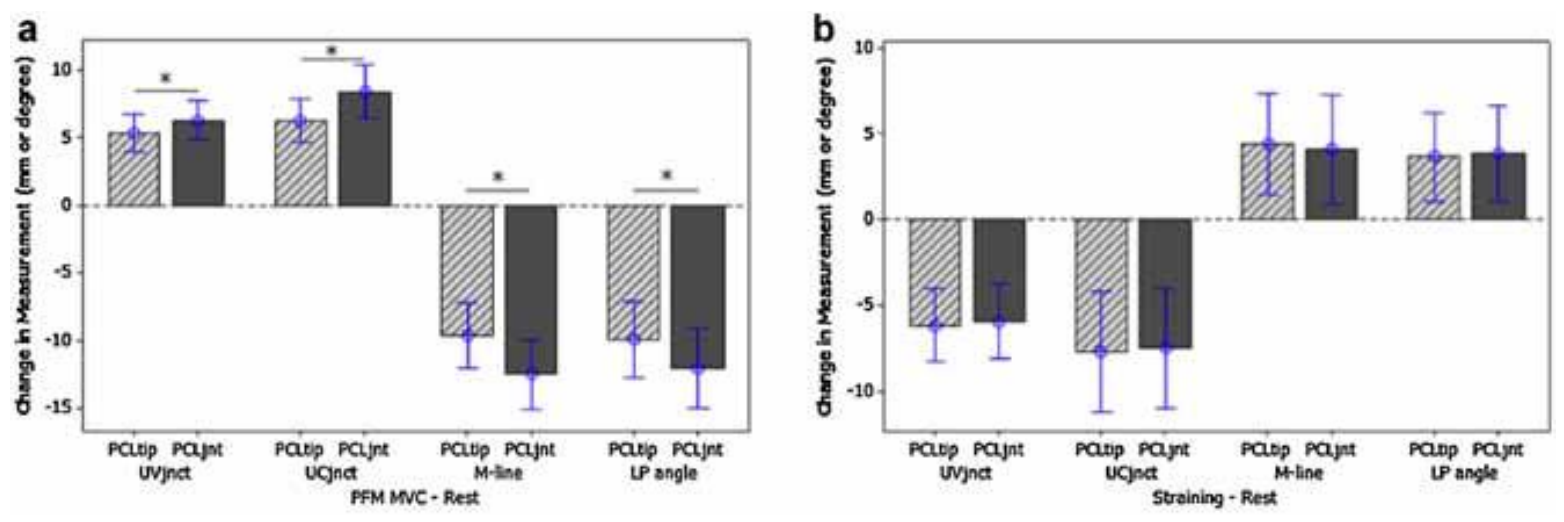

Figure 4.

Changes in the measures of pelvic organ support with (a). A pelvic floor muscle maximum voluntary contraction (PFM MVC) and (b). Straining relative to the two pubococcygeal lines. The zero line indicates the resting measurement; positive values indicate that the measurement increased in size, while negative values indicate a decrease. PCLtip: pubococcygeal line to the tip of the coccyx, PCLjnt: pubococcygeal line to the sacrococcygeal joint, UVjnct (mm): height of the urethrovesical junction, UCjnct (mm): height of the uterovaginal junction, M-line ( $\mathrm{mm}$ ) and LP angle $\left(^{\circ}\right)$ : levator plate angle.

\section{DISCUSSION}

The intra- and inter-rater reliabilities were excellent for both PCLs, indicating that the evaluators were able to produce not only consistent, but also similar measurements. The intra- and inter-rater reliabilities for the other measures of pelvic organ support were good to excellent. However, the intra-class correlations (ICCs) were lower for measurements that had at least one soft tissue landmark, likely because these structures have more rounded contours; hence, consistently positioning the line in the same place is more difficult. The coefficients of variation (CVs) were also higher for the measurements with at least one soft tissue landmark, probably for the same reason described above, and for the shortest measurements. This was likely due to small variations in the measurements, between sets and raters, being proportionally greater to the overall magnitude of the shorter measurements than they were for the longer measurements. The standard errors of the mean (SEMs) indicated a measurement error of not more than $2 \mathrm{~mm}$ (or degrees) for all the measures, suggesting that differences of $2 \mathrm{~mm}$ (or degrees) or greater are true differences. Therefore, a change of at least $2 \mathrm{~mm}$ in the length of the PCLtip was used to define the group demonstrating coccyx movement.

Our results confirm the findings of $\mathrm{B} \emptyset$ et al. $\underline{12}$ that PFM contractions produce movement of the coccyx. These findings are also novel: they not only demonstrated the occurrence of coccyx movement in older women but also established the effect of this movement on commonly used parameters for assessing pelvic organ support. Movement of the coccyx resulted in measurable changes in the length and the position of the PCLtip, subsequently affecting other measures of pelvic organ support that use the PCLtip as a reference line. 
This effect was particularly evident during the PFM MVC. There was less elevation between the rest and PFM MVC conditions in the measures of pelvic organ support that used the PCLtip as their baseline compared to those that used the PCLjnt. This is because the PCLtip was also lifted by the PFM MVC; therefore, these four measurements cannot provide true measures of the lift produced by the PFMs. Further, it suggests that the PCLtip should not be used as a reference line for other measures of pelvic organ support because it may underestimate the effect of the PFM lift on the pelvic organs. It may also mask changes produced by PFM training interventions as PFM training is known to improve the elevation of the PFMs during a PFM MVC.16

Unlike the findings of $\mathrm{B} \emptyset$ et al. 12 there was no difference in the mean length of the PCLtip between the rest and straining conditions. This is because participants demonstrated either a lengthening or a shortening of the PCLtip, likely because those who demonstrated shortening contracted their PFMs during the straining manoeuvre, perhaps in an effort to avoid losing urine or flatus. It is also possible that the standardized straining manoeuvre used in this study was not sufficiently intense to overcome the involuntary PFM contraction associated with increased intra-abdominal pressure.17 A standardized straining manoeuvre was chosen in an effort to avoid variability in effort; however, different levels of effort may be necessary to overcome involuntary PFM contraction in different women. As well, it was assumed that the women would know how to produce a staining effort and, therefore, minimal instructions were given in order to elicit their usual responses. This assumption may have been incorrect as evidenced by the two response patterns. The fact that it is impossible to provide instructions or encouragement to a volunteer while the MRIs are being recorded may also account for the less than maximal effort. Recent research has shown that women must strain for a minimum of $6 \mathrm{sec}$ to achieve maximum pelvic organ descent; $\underline{18}$ in this study the women were required to strain for a minimum of $18 \mathrm{sec}$, sufficient time to perform the manoeuvre. In future research, participants should be specifically instructed on how to produce a maximum straining effort and to relax their PFMs during straining; further, sufficient time to produce maximum pelvic organ descent must also be assured.

Thirteen (28\%) of the women did not produce any movement of the coccyx during either the PFM MVC or straining condition. As movement of the pelvic organs was visible in these women during the tasks and they were able to produce PFM contractions that were confirmed by palpation, dynamometry and electromyography, it is unlikely that the lack of coccyx movement was due to either PFM defects or atrophy or a lack of effort during straining. More probably, the range of motion of the sacrococcygeal and coccygeal joints was limited in these volunteers.19 Since this lack of movement was only observed in a minority of women, it does not change the recommendation that the tip of the coccyx not be used as the posterior landmark for the PCL.

\section{CONCLUSIONS}

A substantial majority of the participants (66\%) demonstrated movement of the coccyx during the PFM MVC and straining. Therefore, to avoid introducing error into measures of pelvic organ support, the PCL should be drawn from the inferior edge of the pubic symphysis to the sacrococcygeal joint, not to the tip of the coccyx.

\section{References}

1 Broekhuis SR, Futterer JJ, et al. A systematic review of clinical studies on dynamic magnetic resonance imaging of pelvic organ prolapse: The use of reference lines and anatomical landmarks. Int Urogynecol J Pelvic Floor Dysfunct 2009; 20: 721-9.

2 El Sayed RF, El Mashed S, et al. Pelvic floor dysfunction: Assessment with combined analysis of static and dynamic MR imaging findings. Radiology 2008; 248: 518-30.

3 Fielding JR. Practical MR imaging of female pelvic floor weakness. Radiographics 2002; 22: 295-304. 
4 Handa VL, Lockhart ME, et al. Magnetic resonance assessment of pelvic anatomy and pelvic floor disorders after childbirth. Int Urogynecol J Pelvic Floor Dysfunct 2009; 20: 133-9.

5 Lockhart ME, Fielding JR, et al. Reproducibility of dynamic MR imaging pelvic measurements: A multiinstitutional study. Radiology 2008; 249: 534-40.

6 Macura KJ, Genadry RR. Female urinary incontinence: Pathophysiology, methods of evaluation and role of MR imaging. Abdom Imaging 2008; 33: 371-80.

7 Yousuf AA, DeLancey JO, et al. Pelvic structure and function at 1 month compared to 7 months by dynamic magnetic resonance after vaginal birth. Am J Obstet Gynecol 2009; 201: 514.e1-7.

8 Hsu Y, Summers A, et al. Levator plate angle in women with pelvic organ prolapse compared to women with normal support using dynamic MR imaging. Am J Obstet Gynecol 2006; 194: 1427-33.

9 Troeger C, Gugger M, et al. Correlation of perineal ultrasound and lateral chain urethrocystography in the anatomical evaluation of the bladder neck. Int Urogynecol J Pelvic Floor Dysfunct 2003; 14: 380-4.

10 Noll L, Hutch JA. The, SCIPP line-an aid in interpreting the voiding lateral cystourethrogram. Obstetr Gynecol 1969; 33: 680-9.

11 Porges R. SCIPP line: Levator ani. Obstet Gynecol 1969; 34: 898-9.

PubMed | CAS | Web of Science®

$12 \mathrm{~B} \emptyset \mathrm{K}$, Lilleås $\mathrm{F}$, et al. Dynamic MRI of the pelvic floor muscles in an upright sitting position. Neurourol Urodyn 2001; 20: 167-74.

13 Shumaker SA, Wyman JF, et al. Health-related quality of life measures for women with urinary incontinence: The Incontinence Impact Questionnaire and the Urogenital Distress Inventory. Continence Program in Women (CPW) Research Group. Qual Life Res 1994; 3: 291-306.

14 Bombieri L, Freeman RM, et al. Objective assessment of bladder neck elevation and urethral compression at colposuspension. Br J Obstet Gynaecol 2002; 109: 395-401.

15 Woodfield CA, Hampton BS, et al. Magnetic resonance imaging of pelvic organ prolapse: Comparing pubococcygeal and midpubic lines with clinical staging. Int Urogynecol J Pelvic Floor Dysfunct 2009; 20: 695-701.

16 Dumoulin C, Peng Q, et al. Changes in levator ani anatomical configuration following physiotherapy in women with stress urinary incontinence. J Urol 2007; 178: 970-7.

17 Junginger B, Baessler K, et al. Effect of abdominal and pelvic floor tasks on muscle activity, abdominal pressure and bladder neck. Int Urogynecol J Pelvic Floor Dysfunct 2010; 21: 69-77.

18 Orejuela F, Shek KL, Dietz HP. editors. The time factor in the assessment of prolapse and levator ballooning. Joint Annual Meeting of the International Continence; Society and International Urogynecological Association; Toronto, Ontario: 2010.

19 Williams A, Newell RLM, Collins P. The back. In: Standring S, editor. Gray's anatomy. 39 edition. London: Elsevier Churchill Livingstone; 2005. p. 733-74. 
"This is the accepted version of the following article: Madill S, Tang A, Pontbriand-Drolet S, Dumoulin C. (2011) Comparison of two methods for measuring the pubococcygeal line from sagittal-plane magnetic resonance imaging. Neurourology and Urodynamics; 30(8):1613-1619., which has been published in final form at: http://onlinelibrary.wiley.com/doi/10.1002/nau.21079/epdf 